\title{
КЛИНИКО-ЛАБОРАТОРНЫЕ ОСОБЕННОСТИ COVID-19 У ГОСПИТАЛИЗИРОВАННЫХ ДЕТЕЙ
}

Мелехина Е.В. ${ }^{1}$, Николаева С.В. ${ }^{1}$, Музыка А.Д. ${ }^{1}$, Понежева Ж.Б. ${ }^{1}$, Ильинская А.С. ${ }^{2}$, Крапивкин А.И. ${ }^{2}$, Солдатова Е.Ю. ${ }^{1}$, Горелов А.В. ${ }^{1,3}$

${ }^{1}$ ФБУН Центральный НИИ Эпидемиологии Роспотребнадзора, Москва, Россия

2 Государственное Бюджетное Учреждение Здравоохранения Города Москвы «Детская Городская Клиническая Больница № 9 им. Г.Н. Сперанского Департамента Здравоохранения Города Москвы» (ГБУЗ «ДГКБ№9 им. Г.Н. Сперанского ДЗМ»), Москва, Россия

3 Первый Московский государственный медицинский университет им. И.М.Сеченова (Сеченовский Университет), г. Москва, Россия

\section{Резюме.}

Цель - выявление основных клинико-лабораторных признаков COVID-19, позволяющих дифференцировать COVID-19 и сезонные ОРИ у детей.

\section{Пациенты и методы.}

Проанализированы истории болезни 44 госпитализированных пациентов (24 мальчиков, 20 девочек) 1-17 лет с симптомами острой респираторной инфекции (ОРИ). Для верификации диагноза определяли PHK SARS-CoV-2 в назофарингеальных мазках (ПЦР), антитела IgM, IgG к SARS-CoV-2 (ИФА), маркеры герпесвирусных, респираторных инфекций, респираторных микоплазмоза и хламидиоза. Статистический анализ проведен с использованием программы IBM SPSS Statistics 26. При расчетах использовали $\mathrm{t}$-критерий Стьюдента, критерий $\mathrm{x}^{2}$ Пирсона, U-критерий Манна-Уитни, точный критерий Фишера. Различия показателей считались статистически значимыми при $\mathrm{p}<0,05$.

\section{Результаты.}

В 1 группу включили 17 детей (39\%), у которых выявили PHK вируса SARSCoV-2 в мазках из ротоглотки, группу сравнения составили 27 детей (41\%), у которых не выявили SARS-CoV-2. Мы определили, что пациенты с ОРИ достоверно чаще жаловались на кашель (p=0,04), а пациенты с COVID-19 - 
на боли в горле ( $\mathrm{p}=0,01)$. Температура одинаково часто повышалась у детей обеих групп. Патология нижних дыхательных путей чаще наблюдалась у пациентов с ОРИ $(\mathrm{p}=0,005)$.

Заключение.

COVID-19 у госпитализированных детей не имеет специфических клинических и лабораторных признаков, что затрудняет дифференциальную диагностику с сезонными ОРИ. Частое выявление сочетанных респираторных инфекций у детей с COVID-19 делает целесообразным проведение скринингового обследования на основные респираторные патогены.

Ключевые слова. COVID-19, дети, респираторные вирусы, сочетанные ОРИ.

Согласно данным литературы, у детей COVID-19 протекает, в целом, благоприятно - бессимптомное течение выявляют примерно у 1/3 пациентов [1]. Приблизительно у половины присутствуют легкие симптомы (такие как лихорадка, усталость, миалгия, кашель) или умеренные симптомы (включая пневмонию с аномальными изображениями грудной клетки). Лишь у 5\% пациентов развиваются более серьезные легочные симптомы (одышка, центральный цианоз, гипоксия); и менее чем $\mathrm{y} 1 \%$ детей отмечено прогрессирование тяжести состояния до критического уровня, которое проявляется как мультисистемный воспалительный синдром (МВС) с острой дыхательной недостаточностью, шоком и/или полиорганной дисфункцией. Тяжесть течения MBC может быть различной - от среднетяжелых форм (с лихорадкой, болью в горле, головной болью, инъекцией конъюнктивы, болью в животе, рвотой, сыпью) до крайне тяжелых - с некротической пневмонией, дисфункцией миокарда, шоком, повреждением почек, аневризмами коронарных артерий и смертью [2]. При MBC, ассоциированном с SARSCov2, наиболее часто поражаются органы желудочно-кишечного тракта, 
сердечно-сосудистой системы, система кроветворения, кожа, слизистые и респираторные органы [3, 4]. Аневризмы коронарных артерий могут выявляться на эхокардиограммах у меньшего количества детей, чем при болезни Кавасаки.

На основании анализа данных по течению COVID-19 у детей в Российской Федерации наиболее частыми симптомами являлись кашель (51\% пациентов), симптомы ринофарингита (25\% пациентов), реже лихорадка (18\% пациентов) [1]. Однако эти данные касались всех случаев COVID-19 у детей, зарегистрированных при первичном амбулаторном обращении за медицинской помощью, включая легкие формы и бессимптомное течение. Вместе с тем дифференциальный диагноз между COVID-19 и сезонными острыми респираторными инфекциями (ОРИ) у пациентов, поступающих в стационар, является важной частью лечебного процесса, так как раннее проведение диагностического поиска и назначение терапии имеет не только важное клиническое, но эпидемиологическое значение. Поэтому целью нашей работы явилось выявление основных клинико-лабораторных п признаков COVID-19, позволяющих дифференцировать COVID-19 и сезонные ОРИ у госпитализированных детей.

\section{Пациенты и методы.}

Проанализированы истории болезни 44 госпитализированных пациентов (24 мальчиков, 20 девочек) в возрасте от 1 до 17 лет, получавших лечение в профильном отделении детской городской клинической больницы №9 им. Сперанского г. Москвы. Отбор пациентов производился методом сплошного скрининга с 1 марта по 30 июня 2020 г. При поступлении в отделение у всех детей были клинические проявления ОРИ. Этиологию ОРИ определяли методами: ПЦР (исследование мазков и/или отделяемого слизистой полости носа, рта, мокроты), ИФА (с определением антител к герпесвирусам 1, 2, 6 типов, цитомегаловирусной, Эпштейна-Барр-вирусной, хламидийной, микоплазменной инфекциям). Диагноз COVID-19 
устанавливали на основании определения PHK вируса SARS-CoV-2 методом ПЦР в назофарингеальных мазках, антител IgM, IgG к SARS-CoV-2 (ИФА) в соответствии с действующими на момент госпитализации детей методическими рекомендациями.

Статистический анализ проводили с использованием программы IBM SPSS Statistics 26. В случае описания количественных показателей, имеющих нормальное распределение, полученные данные объединялись в вариационные ряды, в которых проводился расчет средних арифметических величин (M) и стандартных отклонений (SD), границ 95\% доверительного интервала (95\% ДИ). При сравнении средних величин в нормально распределенных совокупностях количественных данных рассчитывался tкритерий Стьюдента. Совокупности количественных показателей, распределение которых отличалось от нормального, описывались при помощи значений медианы $(\mathrm{Me})$ и нижнего и верхнего квартилей $\left(\mathrm{Q}_{1}-\mathrm{Q}_{3}\right)$. Для сравнения независимых совокупностей в случаях отсутствия признаков нормального распределения данных использовался U-критерий МаннаУитни. Номинальные данные описывались с указанием абсолютных значений и процентных долей. Сравнение показателей, измеренных в номинальной шкале, проводилось при помощи критерия $\chi^{2}$ Пирсона, точного критерия Фишера. Различия показателей считались статистически значимыми при уровне значимости $\mathrm{p}<0,05$.

\section{Результаты и их обсуждение.}

У 17 детей (39\%) выявили PHK вируса SARS-CoV-2 (1, основная группа) (табл. 1). У 12\% (2 детей) 1 группы были выявлены специфические IgG при наличии PHK вируса SARS-CoV-2 в мазках из носоглотки, а у 6\% (1 ребенок) - PHK вируса и специфические IgM, IgG. У 27 детей PHK SARSCoV-2 и IgM выявлены не были, эти дети вошли во 2 группу (группу сравнения). Изолированное обнаружение специфических IgG при отсутствии выделения PHK вируса SARS-CoV-2 методом ПЦР из носоглотки у 1 (7\%) 
ребенка связано, по-видимому, с перенесенным в бессимптомной форме COVID-19, что не было диагностировано ранее (табл. 1).

Таблица 1. Диагностика COVID-19 у наблюдаемых детей

\begin{tabular}{|c|c|c|c|c|}
\hline \multirow{3}{*}{$\begin{array}{c}\text { Методы } \\
\text { исследовани } \\
\text { я }\end{array}$} & \multicolumn{4}{|c|}{ Группы пациентов } \\
\hline & \multicolumn{2}{|c|}{$\begin{array}{l}\text { COVID-19 (+) } \\
(n=17)\end{array}$} & \multicolumn{2}{|c|}{$\begin{array}{c}\text { ОРИ } \\
(\mathrm{n}=27)\end{array}$} \\
\hline & $\begin{array}{c}\text { Обследован } \\
\text { о }\end{array}$ & $\begin{array}{c}\text { Положительны } \\
\text { й результат }\end{array}$ & $\begin{array}{c}\text { Обследован } \\
\text { о }\end{array}$ & $\begin{array}{c}\text { Положительны } \\
\text { й результат }\end{array}$ \\
\hline $\begin{array}{c}\text { ПЦР (PHK } \\
\text { SARS-CoV- } \\
\text { 2) }\end{array}$ & 17 & 17 & 27 & 0 \\
\hline ИФА (IgM) & 12 & 2 & 3 & 0 \\
\hline ИФА (IgG) & 12 & 3 & 3 & 1 \\
\hline
\end{tabular}

Возрастной состав включенных в исследование был следующим: дети от 1 года до 7 лет - 41\% (18), дети старше 7 лет - 59\% (26), сравниваемые группы были сопоставимы по полу и возрасту. 71\% госпитализированных детей приходилось на школьников (различия статистически достоверны, $\mathrm{p}=0,02)$ (рис. 1). 


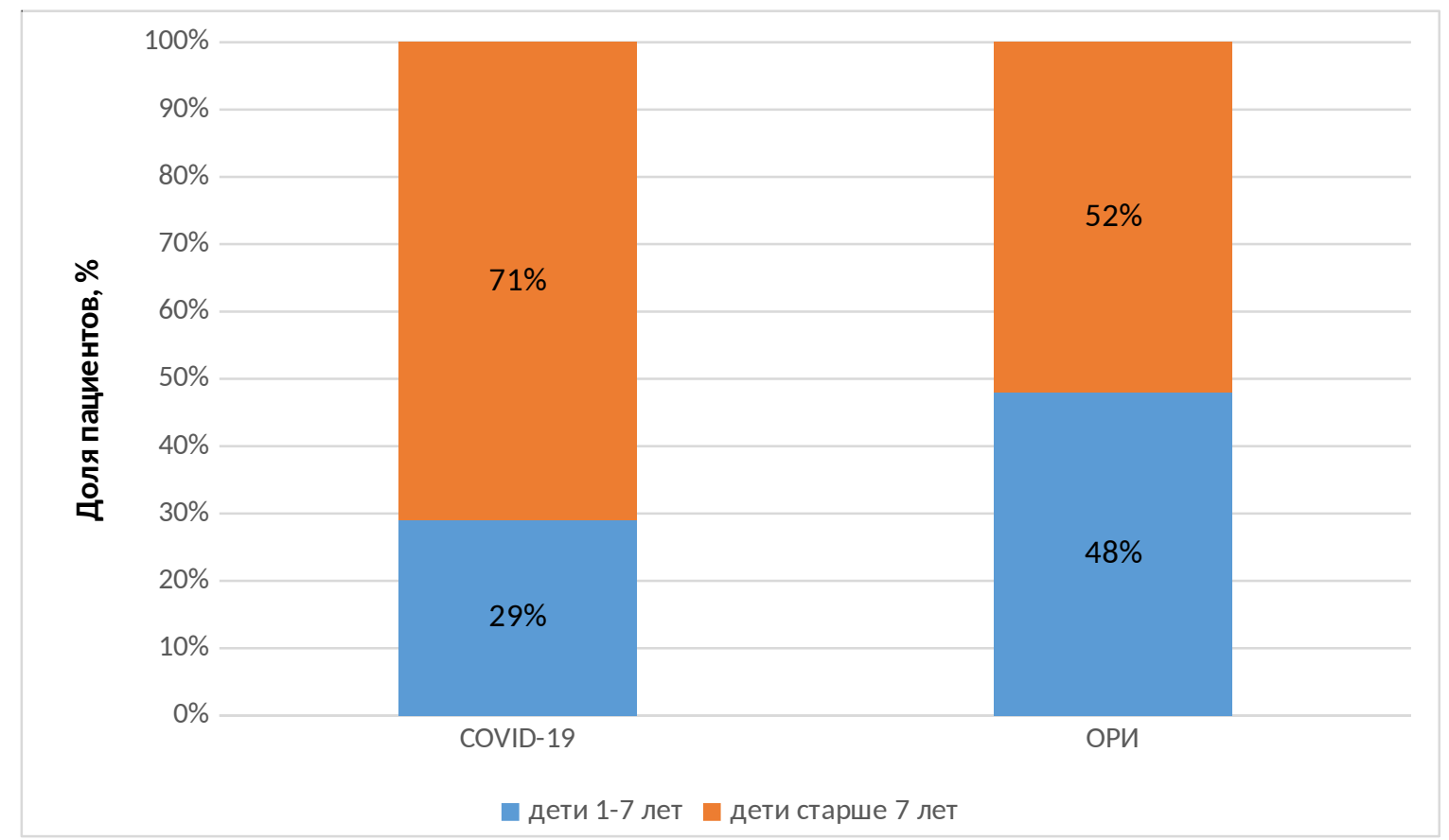

Рис. 1. Возрастная характеристика групп (в \%).

В гендерной структуре госпитализированных детей незначительно преобладали мальчики - 55\%. В 1 группе мальчиков было 59\% (10/17), девочек - 41\% (7/17), в группе сравнения мальчиков и девочек было 52\% и $48 \%$, соответственно.

Наиболее частыми симптомами у детей при поступлении в стационар были повышение температуры (84\%) и кашель (66\%). Лихорадка имела место y 82\% детей с COVID-19 и 85\% детей без COVID-19, при этом у большинства детей отмечали фебрильную температуру. Кашель чаще наблюдался у пациентов с ОРИ $(\mathrm{p}=0,04)$ (между сопоставляемыми признаками отмечалась средняя связь $(\mathrm{V}=0,353))$ (таблица 2). На боли в горле чаще жаловались дети с COVID-19 (p=0,01) (между сопоставляемыми признаками отмечалась относительно сильная связь $(\mathrm{V}=0,421))$. Другие симптомы: интоксикация 
(проявляющаяся в слабости), проявления ринита, боли в животе, нарушения стула встречались у детей обеих групп одинаково часто.

Таблица 2. Жалобы при поступлении в стационар

\begin{tabular}{|c|c|c|c|c|c|}
\hline \multirow[t]{2}{*}{ Симптомы } & \multicolumn{2}{|c|}{$\begin{array}{l}\text { COVID-19 }(+), \\
n=17\end{array}$} & \multicolumn{2}{|c|}{ ОРИ, $\mathrm{n}=27$} & \multirow[t]{2}{*}{$\mathrm{p}$} \\
\hline & Абс. & $\%$ & Абс. & $\%$ & \\
\hline Кашель & 9 & $53 \%$ & 23 & $85 \%$ & $0,04^{*}$ \\
\hline Слабость & 6 & $35 \%$ & 7 & $26 \%$ & 0,52 \\
\hline Заложенность носа & 6 & $35 \%$ & 7 & $26 \%$ & 0,52 \\
\hline Отделяемое из носа & 5 & $29 \%$ & 5 & $19 \%$ & 0,47 \\
\hline Боль в горле & 6 & $35 \%$ & 1 & $4 \%$ & $0,01^{*}$ \\
\hline $\begin{array}{c}\text { Боль в животе, } \\
\text { нарушения стула }\end{array}$ & 4 & $24 \%$ & 3 & $11 \%$ & 0,40 \\
\hline Боль в ухе & 0 & $0 \%$ & 3 & $11 \%$ & 0,66 \\
\hline Головная боль & 2 & $12 \%$ & 0 & $0 \%$ & 0,14 \\
\hline Аносмия & 2 & $12 \%$ & 0 & $0 \%$ & 0,14 \\
\hline Сыпь & 1 & $6 \%$ & 0 & $0 \%$ & 0,39 \\
\hline Дизурия & 1 & $6 \%$ & 0 & $0 \%$ & 0,39 \\
\hline \multicolumn{6}{|l|}{ Температура } \\
\hline Нормальная & 3 & $18 \%$ & 4 & $15 \%$ & 1,0 \\
\hline $37,0-38,0$ & 4 & $24 \%$ & 5 & $19 \%$ & 0,72 \\
\hline $38,1-40,0$ & 10 & $59 \%$ & 18 & $67 \%$ & 0,75 \\
\hline
\end{tabular}

По результатам физикального исследования пациентов при поступлении в инфекционное отделение достоверных различий в группах не получено, однако аускультативные изменения в легких (ослабленное дыхание, хрипы) несколько чаще наблюдались у пациентов с ОРИ, зернистость задней стенки глотки - напротив, у пациентов с COVID-19.

Не выявлено статистически значимых изменений лабораторных показателей у детей с COVID-19. Те или иные сдвиги в клиническом и биохимическом анализах крови, выявленные у большинства 
госпитализированных детей, были несущественными и быстро обратимыми (табл. 3).

Таблица 3. Характеристика гематологических показателей у госпитализированных детей.

\begin{tabular}{|c|c|c|c|c|c|c|c|}
\hline & \multicolumn{2}{|c|}{ ВСЕГО, n=44 } & \multicolumn{2}{|c|}{$\begin{array}{c}\text { COVID-19 (+), } \\
\mathrm{n}=17\end{array}$} & \multicolumn{2}{|c|}{ ОРИ, $\mathrm{n}=27$} & \multirow{2}{*}{$\mathbf{p}$} \\
\hline & $\mathrm{M} \pm \mathrm{SD}$ & $\begin{array}{l}95 \% \\
Д И\end{array}$ & $\mathrm{M} \pm \mathrm{SD}$ & $\begin{array}{c}95 \% \\
\text { ДИ }\end{array}$ & $\mathrm{M} \pm \mathrm{SD}$ & $\begin{array}{l}95 \% \\
\text { ДИ }\end{array}$ & \\
\hline \multicolumn{8}{|c|}{ Клинический анализ крови } \\
\hline $\begin{array}{c}\text { Эритроцит } \\
\text { ы, *10 } 12 \\
\text { клеток }\end{array}$ & $\begin{array}{c}4,7 \pm 0,0 \\
7\end{array}$ & $\begin{array}{c}4,56- \\
4,83\end{array}$ & $\begin{array}{c}4,71 \pm 0 \\
08\end{array}$ & $\begin{array}{c}4,53- \\
4,89\end{array}$ & $\begin{array}{c}4,69 \pm 0 \\
1\end{array}$ & $4,49-4,9$ & $\begin{array}{l}>0,0 \\
5\end{array}$ \\
\hline $\begin{array}{c}\text { Гемоглобин } \\
\text {, г/л }\end{array}$ & $\begin{array}{c}134,6 \pm 2 \\
, 46\end{array}$ & $\begin{array}{l}129,6- \\
139,6\end{array}$ & $\begin{array}{c}137,31 \pm \\
3,75\end{array}$ & $\begin{array}{l}129,22- \\
145,41\end{array}$ & $\begin{array}{c}132,7 \pm 3 \\
, 27\end{array}$ & $\begin{array}{l}125,84- \\
139,55\end{array}$ & $\begin{array}{c}>0,0 \\
5\end{array}$ \\
\hline $\begin{array}{c}\text { Тромбоцит } \\
\text { ы, *10 } \\
\text { клеток } \\
\end{array}$ & $\begin{array}{c}252,53 \pm \\
13,53\end{array}$ & $\begin{array}{l}225,01- \\
280,05\end{array}$ & $\begin{array}{c}233,29 \pm \\
20,17\end{array}$ & $\begin{array}{l}189,71- \\
276,86\end{array}$ & $\begin{array}{c}266 \pm 17 \\
95\end{array}$ & $\begin{array}{l}228,43- \\
303,57\end{array}$ & $\begin{array}{c}>0,0 \\
5\end{array}$ \\
\hline $\begin{array}{l}\text { Лейкоциты, } \\
\text { *10 } 10^{9} \text { клеток }\end{array}$ & $\begin{array}{c}8,07 \pm 0, \\
66 \\
\end{array}$ & $\begin{array}{c}6,74- \\
9,41 \\
\end{array}$ & $\begin{array}{c}7,21 \pm 1 \\
07\end{array}$ & $\begin{array}{l}4,89- \\
9,52 \\
\end{array}$ & $\begin{array}{c}8,68 \pm 0 \\
82 \\
\end{array}$ & $\begin{array}{l}6,96- \\
10,4 \\
\end{array}$ & $\begin{array}{c}>0,0 \\
5\end{array}$ \\
\hline $\begin{array}{c}\text { Нейтрофил } \\
\text { ы, \% }\end{array}$ & $\begin{array}{c}48,6 \pm 2 \\
86\end{array}$ & $\begin{array}{l}42,81- \\
54,46\end{array}$ & $\begin{array}{c}45,13 \pm 4 \\
, 55\end{array}$ & $\begin{array}{l}35,3- \\
54,96\end{array}$ & $\begin{array}{c}51,1 \pm 3 \\
67\end{array}$ & $\begin{array}{l}43,4- \\
58,79\end{array}$ & $\begin{array}{c}>0,0 \\
5\end{array}$ \\
\hline $\begin{array}{c}\text { Эозинофил } \\
\text { ы, \% }\end{array}$ & $\begin{array}{c}2,69 \pm 0 \\
41\end{array}$ & $\begin{array}{l}1,86- \\
3,53\end{array}$ & $\begin{array}{c}3,59 \pm 0 \\
83\end{array}$ & $\begin{array}{c}1,79- \\
5,38\end{array}$ & $\begin{array}{c}2,07 \pm 0 \\
34\end{array}$ & $\begin{array}{c}1,36- \\
2,78\end{array}$ & $\begin{array}{c}>0,0 \\
5\end{array}$ \\
\hline $\begin{array}{c}\text { Лимфоциты } \\
\text {, \% }\end{array}$ & $\begin{array}{c}34,66 \pm 2 \\
, 61\end{array}$ & $\begin{array}{l}29,34- \\
39,98\end{array}$ & $\begin{array}{c}36,63 \pm 4 \\
, 53\end{array}$ & $\begin{array}{c}26,85- \\
46,42\end{array}$ & $\begin{array}{c}33,29 \pm 3 \\
, 18\end{array}$ & $\begin{array}{l}26,63- \\
39,94\end{array}$ & $\begin{array}{c}>0,0 \\
5\end{array}$ \\
\hline $\begin{array}{c}\text { Моноциты, } \\
\%\end{array}$ & $\begin{array}{c}11,48 \pm 0 \\
, 86\end{array}$ & \begin{tabular}{|l|}
$9,73-$ \\
13,23 \\
\end{tabular} & $\begin{array}{c}11,6 \pm 1 \\
42\end{array}$ & $\begin{array}{l}8,52- \\
14,67 \\
\end{array}$ & $\begin{array}{c}11,4 \pm 1 \\
1\end{array}$ & $\begin{array}{l}9,09- \\
13,71 \\
\end{array}$ & $\begin{array}{c}>0,0 \\
5\end{array}$ \\
\hline \multicolumn{8}{|c|}{ Биохимический анализ крови } \\
\hline & $\mathrm{Me}$ & $\mathrm{Q}_{1}-\mathrm{Q}_{3}$ & $\mathrm{Me}$ & $\mathrm{Q}_{1}-\mathrm{Q}_{3}$ & $\mathrm{Me}$ & $\mathrm{Q}_{1}-\mathrm{Q}_{3}$ & \\
\hline АЛТ, ед/л & 17 & $13-21$ & 16 & $11,5-22$ & 17 & $13-21$ & $\begin{array}{l}>0,0 \\
5\end{array}$ \\
\hline АСТ, ед/л & 35 & $26-45$ & 32 & $24-46,5$ & 35,5 & $26-45$ & $\begin{array}{c}>0,0 \\
5\end{array}$ \\
\hline $\begin{array}{c}\text { Общий } \\
\text { белок, г/л }\end{array}$ & 68,1 & $64-73$ & 69,2 & $\begin{array}{c}64,55- \\
74,1\end{array}$ & 67,45 & $\begin{array}{l}63,9- \\
71,1\end{array}$ & $\begin{array}{c}>0,0 \\
5\end{array}$ \\
\hline $\begin{array}{l}\text { Мочевина, } \\
\text { ммоль/л }\end{array}$ & 3,7 & $2,9-4,2$ & 4,1 & $3,3-4,75$ & 3,6 & $2,8-4,1$ & $\begin{array}{c}>0,0 \\
5\end{array}$ \\
\hline $\begin{array}{l}\text { Креатинин, } \\
\text { ммоль/л }\end{array}$ & 45,9 & $\begin{array}{l}37,19- \\
52,92\end{array}$ & 46,52 & $\begin{array}{c}41,34- \\
56,99\end{array}$ & 42,57 & $\begin{array}{c}36,54- \\
51,7\end{array}$ & $\begin{array}{c}>0,0 \\
5\end{array}$ \\
\hline $\begin{array}{c}\text { Билирубин } \\
\text { общий, } \\
\text { мкмоль/л }\end{array}$ & 6,4 & $4,9-10,4$ & 6,5 & $\begin{array}{l}5,45- \\
7,85\end{array}$ & 6,15 & $4,5-10,6$ & $\begin{array}{c}>0,0 \\
5\end{array}$ \\
\hline
\end{tabular}




\begin{tabular}{|c|c|c|c|c|c|c|c|}
\hline $\begin{array}{c}\text { Глюкоза, } \\
\text { ммоль/л }\end{array}$ & 4,34 & $\begin{array}{c}4,01- \\
4,72\end{array}$ & 4,31 & $\begin{array}{c}3,74- \\
4,85\end{array}$ & 4,4 & $\begin{array}{c}4,11- \\
4,63\end{array}$ & $\begin{array}{c}>0,0 \\
5\end{array}$ \\
\hline СРБ, мг/л & 9,3 & $1,3-34,2$ & 1,9 & $\begin{array}{c}0,6- \\
36,55\end{array}$ & 12,3 & $2,6-30,7$ & $\begin{array}{c}>0,0 \\
5\end{array}$ \\
\hline
\end{tabular}

Поражение верхних дыхательных путей (ВДП) имело место у 55\% пациентов, нижних дыхательных путей (НДП) с развитием пневмонии или бронхита - у 45\% (рис. 2), при этом патология НДП чаще наблюдалась у пациентов с ОРИ (p=0,005), между сопоставляемыми признаками отмечалась относительно сильная связь $(\mathrm{V}=0,443)$.

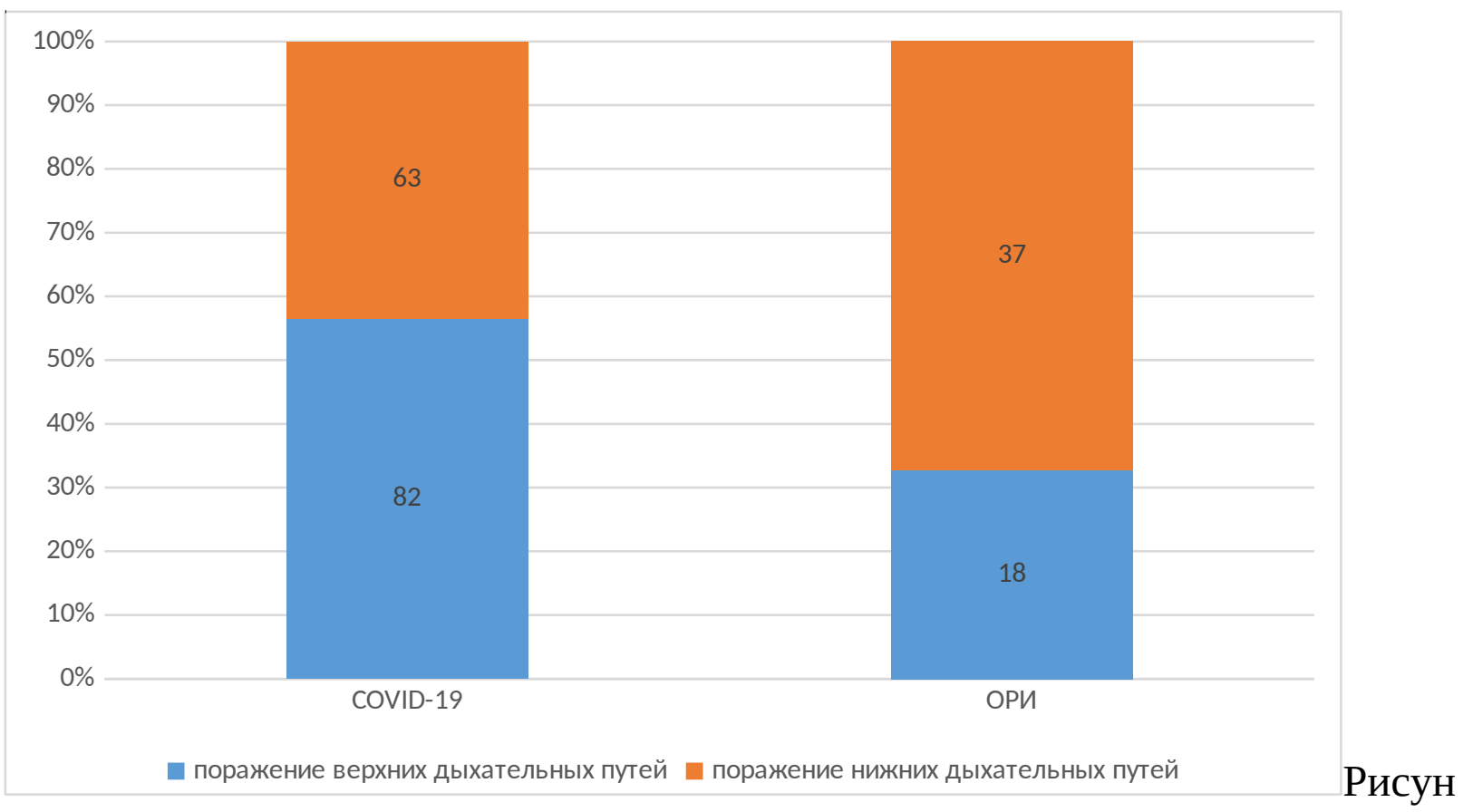

ок 2. Уровень поражения респираторного тракта в сравниваемых группах (в $\%)$.

Сочетанное инфицирование выявили в 27\% всех случаев. В 1 группе сочетанное инфицирование SARS-CoV-2 с другим респираторным патогеном регистрировали у 29\% детей. Среди микст-форм отмечено сочетание SARS- 
CoV-2 + M. pneumoniae (18\%), SARS-CoV-2 + герпесвирусные инфекции (ГВИ) (18\%), SARS-CoV-2 + Chl. pneumoniae (6\%). У пациентов с ОРИ чаще выявляли M. pneumoniae (различия статистически не значимы), что, повидимому, и способствовало более частому поражению НДП с соответствующими аускультативными изменениями в легких (ослабленное дыхание, хрипы) у детей этой группы.

Таким образом, выявленные клинические особенности течения ОРИ у детей (более выраженный кашлевой синдром, менее выраженное поражение ротоглотки и частые аускультативные изменения в легких) по сравнению с пациентами, переносящими COVID-19, можно объяснить особенностями этиологии ОРИ, характерных для настоящего периода, в котором преобладающими патогенами были SARS-CoV-2 и M. Pneumoniae, а также их сочетания.

Заключение.

Среди пациентов с COVID-19 преобладали школьники, у которых отмечалось поражение ВДП. В дебюте болезни характерными клиническими симптомами у таких детей были боли в горле. У детей, госпитализированных с ОРИ, было характерно развитие кашля, аускультативных изменений в легких, поражение НДП. Отмечено, что выявление во время пандемии COVID-19 в мазках из носоглотки вируса SARS-CoV-2 не исключает заражения другими респираторными патогенами. Поскольку в осеннезимний сезон регистрируются пиковые подъемы гриппа и сезонных респираторных инфекций, важно учитывать необходимость скрининга на основные респираторные патогены, принимая во внимание возможность инфицирования не только COVID-19.

Литература.

1. Горелов А.В., Николаева С.В., Акимкин В.Г. Коронавирусная инфекция COVID-19 у детей в Российской Федерации. 
Инфекционные болезни. 2020; 18(3): 15-20. DOI: 10.20953/17299225-2020-3-15-20.

2. Nakra NA, Blumberg DA, Herrera-Guerra A, Lakshminrusimha S. MultiSystem Inflammatory Syndrome in Children (MIS-C) Following SARSCoV-2 Infection: Review of Clinical Presentation, Hypothetical Pathogenesis, and Proposed Management. Children (Basel). 2020;7(7):69. Published 2020 Jul 1. doi:10.3390/children7070069

3. Whittaker E, Bamford A, Kenny J, et al. Clinical characteristics of 58 children with a pediatric inflammatory multisystem syndrome temporally associated with SARS-CoV-2. JAMA. 2020; 324:259-269.

4. Feldstein LR, Rose EB, Horwitz SM, et al. Multisystem inflammatory syndrome in U.S. children and adolescents. $\mathrm{N}$ Engl $\mathrm{J}$ Med. 2020;383:334-346.

5. Е.А. Дондурей, Л.Н. Исанкина, О.И. Афанасьева, А.В. Титёва, Т.В. Вишневская, В.А. Кондратьев, И.А. Грязнова, М.В. Березина, М.А. Золотова, B.M. Волжанин. Характеристика COVID-19 у детей: первый опыт работы в стационаре Санкт-Петербурга. Журнал инфектологии. 2020; 12(3): 56-63. DOI: 10.22625/2072-6732-2020-123-56-63. 